\title{
Deep Learning for Image Based Mango Leaf Disease Detection
}

\author{
Sampada Gulavnai, Rajashri Patil
}

\begin{abstract}
Among world's mango producing countries, India ranks first and account $50 \%$ of the world's mango production. The mango fruit is popular because of its wide range of adaptability, high nutritional value, different variety, delicious taste and excellent flavor. The fruit contains vitamin $A$ and vitamin $C$ in a rich extent. The crop is prone to diseases like powdery mildew, anthracnose, die back, blight, red rust, sooty mould, etc. Disorders may also impact the plant in the absence of effective case and control measures. These include change of form, biennial bearing, fall of fruit, black top, clustering, etc. The farmer must consult and take professional support for the prevention / control of diseases and crop disorder. New techniques of detecting mango disease are required to promote better control to avoid this crisis. By considering this, paper describes image recognition which provides cost effective and scalable disease detection technology. Paper further describes new deep learning models which give an opportunity for easy deployment of this technology. By considering a dataset of mango disease, pictures are taken from Konkan area in India. Transfer learning technique is used to train a profound Convolutionary Neural Network (CNN) to recognize $91 \%$ accuracy.
\end{abstract}

Keywords: Crop, Mango, Neural Network, deep learning, Image Recognition, Convolutionary Neural Network (CNN).

\section{INTRODUCTION}

Mango is known as 'The King of Fruits' is one of the major fruit crops cultivated in various countries in the world. India produces $40 \%$ of mangoes production and stands first in the mango growing countries in the world [1]. Crop yield is affected by Pests and diseases and kill about $30 \%$ to $40 \%$ of the crop yield [2]. The recognition of mango plant infections is finished by unaided eye perception, which gives less exactness. The various diseases affecting mango plant are not recognized by the farmers which cause less production of mango fruits. Mango crop is greatly affected by various diseases. The disease creates unevenly shaped black patches. These patches appear over surface of the leaf or early grown fruits [3]. These patches begin in tiny form, but quickly they spread to the entire fruit or leaves and fruits rot as a result. These kinds of illnesses need to be detected and controlled in a particular period of time at their original stage. Therefore, it is important to kill these diseases before affecting any basic activity of mango plant body such as photosynthesis, transpiration, pollination, fertilization, germination, etc. Pathogens such as champignons, bacteria and viruses cause these diseases.

Revised Manuscript Received on November 11, 2019.

* Correspondence Author

Dr.Mrs.SampadaGulavnai, Asso. Prof., Bharati Vidyapeeth (Deemed to be University), Pune , Inst. of Management, Kolhapur, India.

Prof. Ms .Rajashri Patil, Asso. Prof., Vivekanand College, Kolhapur, India
For this, farmers need to monitor the plant body continuously which is a time-consuming method. Some technique is required for the early detection of disease in the plant. Early recognition of disease in the field is the initial step in managing the detection and spread of mango diseases. Traditional approaches to disease identification depend on the assistance from agricultural organizations, but these methods are restricted due to low ability for logistics and human infrastructure.

By using technology for internet penetration, smart phone and unmanned aerial vehicle (UAV) offers fresh instruments for disease detection depends upon automated image identification which help quick detection in large scale. Previous study has shown that plant disease recognition in wheat is automated with image recognition, Apples [4][5][6] and other healthy and disease affected plants[7]. Detection of mango leaf diseases based on automated picture recognition by extraction of features has shown promising outcomes [8]. But extraction characteristics are computationally intensive and require solid performance expertise. In order to capitalize on smart phone technology, models need to be quick and tailored to a restricted processing power. In transfer learning a model is to be trained for a big image dataset is retrained for newly derived classes, provides a short way to deep learning models due to less computational demands. Here we explored the possibility of adjusting an already qualified neural network model using deep learning to identify the measure of disease occurrence using an infield dataset of pictures (app. 8853) consisting of four diseases.

\section{METHODS OF DATA COLLECTION}

i) The Mango Image Dataset:

In experimental areas belonging to the 'Regional Fruit Research Center', Vengurla and Mango Research Center Rameshwar, Tal. Devgad, Dist. Sindhudurga ,India. The mango leaf pictures were taken with a frequently accessible sony digital camera and mobile camera. To construct the first dataset, the entire mango leaf was photographed. Approximately 3500 pictures were taken over a two-week period. Pictures of ailments were taken utilizing a few genotypes of mango to provide the deep learning model with the complete spectrum of symptoms for each disease. Each disease or type of pest harm was unique and the variety of symptom expression in species was less than the contrasts between diseases.

Images for co-infections were tested to restrict the amount of pictures with various diseases. This dataset is termed as the "original mango dataset" comprised 8,853 images. The pictures were then transformed using data augmentation techniques through python coding into separate images to create the secondary dataset. This dataset is referred as the 
"Mango leaflet dataset," comprised $8852 \mathrm{~V}$ images of mango leaves. Fig. 1 shows examples from datasets like mango dataset S1-S4 show examples for each mango disease. These datasets were screened to focus on model efficiency with entire leaf pictures, but some pictures compared to more cropped leaves. The fundamental hypothesis was that the images of cropped leaves (leaflet mango data set) would enhance model efficiency in order to properly recognize a disease as the data set was greater. we assumed that by concentrating on symptomatic images, the end consumers would attempt to find a diagnosis for a disease. These datasets included four distinct classes manually allocated by MRC mango disease specialists based on in-field diagnosis. To evaluate the efficiency of the model. , for all classes, we used the images as they are and with different field backgrounds. The four classes include four types of diseases. The image count in the original dataset of each class: mango anthracnose disease (1952 images), mango powdery mildew disease (1217 images), red rust (3479 images) and mango golmich (2205 images).
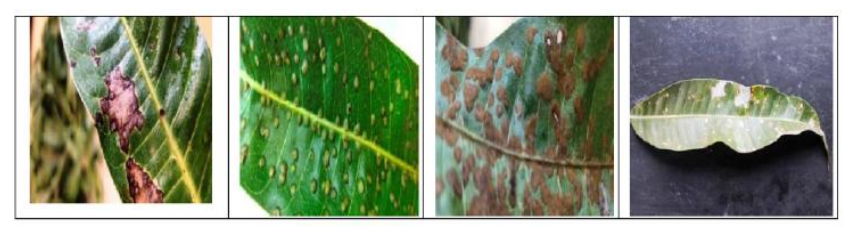

Figur 1:(a) Anthracnose (b)Golmich(c) Red Rust(d) and powdery mildew .

ii)The diseases and pest class symptoms observed :

a)The Powdery Mildew: The symptoms of this disease is the growth of white surface powdery fungal on panicle stalks, flowers ,young fruits and the roots. The flowers and fruits affected fall and significantly reduce the plant load or even stop the fruit collection. All the parts of the inflorescence, leaves and fruits get parasitized by young tissues of fungus. Young leaves are struck on both sides, but on the grower surface it is more prominent. Such patches also coalesce and cover larger areas which turn into colored purple gray.

b)Anthracnose: The disease causes signs of leaf blot, flower blight, wither top, twig blight and fruitrot.Tender shoots and leaves are easily affected, causing young branches to ' die back '. Older branches can also be contaminated with wounds that can be fatal in severe cases. Blossom blight can vary in severity from mild to severe panicle infection depending on the prevailing weather conditions.Black spots form both on the fruit and on the panicles. Severe infection kills the whole inflorescence, leading to no fruit environment. Infected young fruits grow black spots, shrink and fall. Infected fruit at maturity stores the champignon and causes significant losses during processing, transit and marketing.

c)Red Rust : The pathogens replicate and live in affected patches of leaves and stems. In mango growing areas, an algae-induced red rust disease was observed. The rusty red spots can easily recognize the disease, mostly on the leaves and sometimes on the young branches ' petioles and bark. The spots are painted greenish gray and textured velvety and further turned into reddish brown. Sometimes the circular and slightly elevated spots converted into larger and irregular spots. In closely planted orchards, the disease is more common.
d)Golmich: In this disease mango fruits, blossom, leaves, panicles, twigs and bark of stem get affected by the scab fungus. Affected area mostly find in circular shape, slightly angular, elongated style with $2-4 \mathrm{~mm}$ in diameter, brown and in during rainy season, lesions appear indifferent size, shape and color.

\section{CONVOLUTIONAL NEURAL NETWORKS (CNN) MODEL:}

The transfer learning is used in a proposed Convolutional Neural Networks (CNN) model for the mango images dataset. CNN is leading soft computing approach in computer vision tasks [9]. CNN learn characteristics from hierarchy of image pixels to create classifiers and train the layers together as compared to traditional methods of training classifiers with existing feature extraction techniques. Because of the complexity of the model, CNN takes small time span to weeks to train complete model. Transferring learning is applied is used as a model practiced by adopting a fully trained model for a set of image classes and retraining existing weights for new classes. In proposed model the current weights of the ResNet CNN system were retrained to identify the mango image datasets using the large amount of visual information already gained by ResNet. Previous research found that learning transfer is successful for a variety of applications $[10,7]$ and the computational requirements are much lower than learning from scratch, which supports mobile applications. It has much lower computational requirements, which is a beneficial to develop various types of mobile applications. The performance of training the final layer of the CNN model ResNet for the new mango image datasets with three architectures: ResNet 18, ResNet 34 and ResNet51 is measured. The latest version of the ResNet was implemented in FastAI. ResNet 51 is 51 layers deep, but the computation cost is only 2.5 times higher than that of ResNet 34 with 34 layers and ResNet 18 with 18 layers. Starting with the ResNet model, several design principles are implemented to expand convolutionary networks to improve performance with a little bit increase in computational costs and devices like mobile phones and drones with limited memory and less computational power get a significant benefits.

\section{PERFORMANCE MEASUREMENTS}

With different sizes of mango image classes and different $\mathrm{CNN}$ architectures, the researchers measured and achieved results with less deviations so as to perform a robust validation and test. The experiments were carried out with different ranges of training and validation dataset splits. For each experiment the overall accuracy is reported as the number of samples in all classes that were different.

\section{RESULTS}

The overall accuracy for the augmented mango dataset is measured with ResNet18, ResNet34 and ResNet50. ResNet18 CNN architecture gives 91\% accuracy with validation set of $15 \%$, ResNet34 CNN architecture gives the accuracy is $90.88 \%$ with validation set of $15 \%$ and ResNet50 provides the accuracy of $91.50 \%$. 


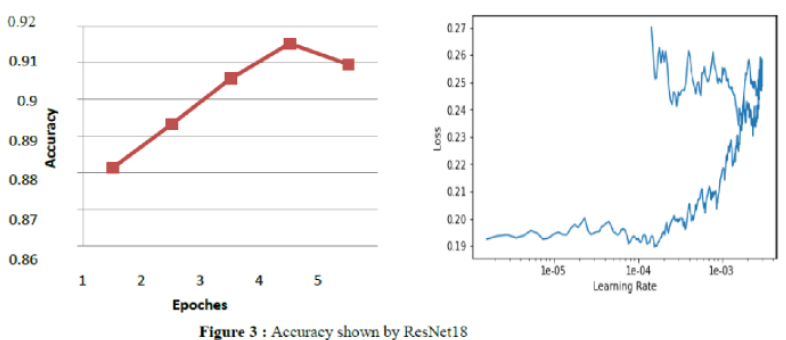

- Fig. 3 shows ResNet18 CNN architecture no. of epochs and its accuracy for $15 \%$ validation set and its learning rate. The time varies from 1.17 seconds to 1.21 seconds for each epoch.

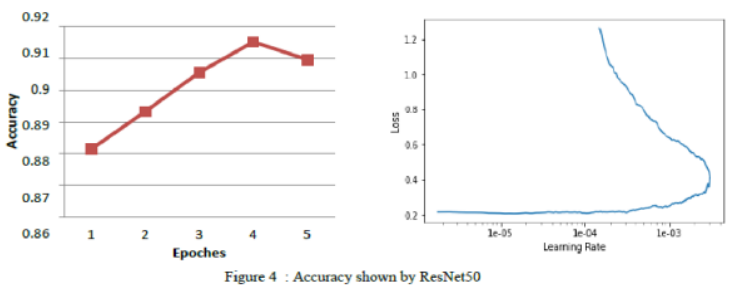

- Fig. 4 shows the accuracy shown by ResNet50 architecture with its learning rate. The time varies from 2.29 to 2.44 seconds.

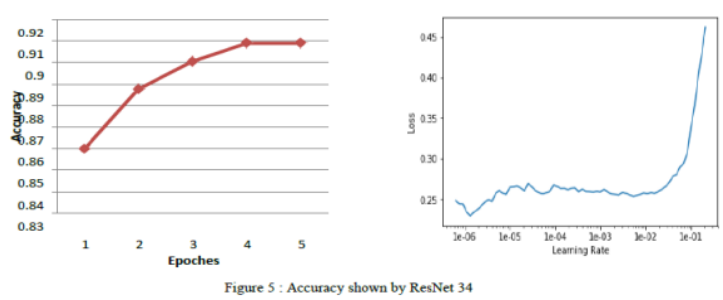

- Fig. 5 shows the accuracy for ResNet34 architecture with $20 \%$ validation set. The time varies from 1.11 to 1.22 seconds for each epoch.

- All models used here analyze better than randomly guessing, even with the images are having different background with the objects like human hands, soil or various distracting things. The Results shows that the models were not over fitted to the datasets because the split training validation information had a tiny impact on the provided general accuracies .

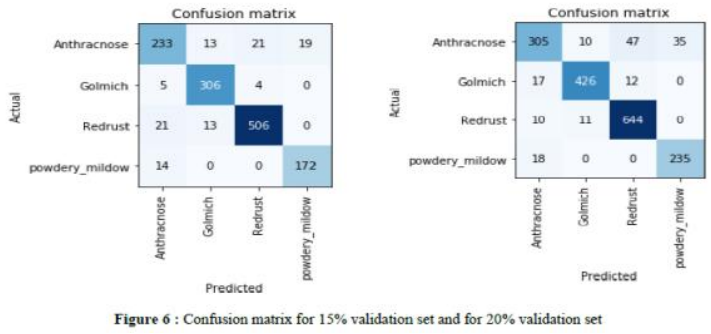

- The confusion matrix shown in Figure 6 from the mango dataset allows a more deep analysis by showing how the model performance varies with different disease identified in the images. In the first confusion matrix plot for the $15 \%$ validation set, data split towards the rows shows the true classes and the column shows the predicted classes. The diagonal cell reflects the proportion of instances that the qualified network correctly predicts the groups of observations. It shows the proportion that fits towards real and expected classes. The off-diagonal cells reflect where there were inconsistencies in the network.
- The proportion shown in the off-diagonal cells and on diagonal cells of the models displayed the highest reported prediction accuracy is 0.95 for golmich, when $20 \%$ validation set is taken for ResNet18 architecture and redrust is 0.95 when $15 \%$ validation set is taken for ResNet50.

\section{DISCUSSION AND CONCLUSION}

The findings of this research indicate that dieasse identification from image with the convolutionary neural network ResNet50 is a strong technique for high precision automated identification of mango disease. This technique prevents the complicated image extraction and a model can be trained on machines and can be implemented for mobile devices. In this study three $\mathrm{CNN}$ architectures are used for four different classes of mango diseases. Therefore, this study shows that deep learning neural network provides a strong channel for in-field disease detection using convolutionary neural networks using an object dataset, and is a powerful technique for high precision automatic mango disease identification.

\section{FUTURE WORK}

The best model is to be developed for the mobile devices using the best $\mathrm{CNN}$ architecture to monitor mango diseases in India.

\section{REFERENCES}

1. Mango Leaf Diseases Identification Using Convolutional Neural Network S. Arivazhagan1, S.Vineth Ligi2 Center for Image Processing and Pattern Recognition, Department of ECE,1,2Mepco Schlenk Engineering College, Sivakasi. August 4, 2018

2. http://www.sciencedirect.com/topics/agricultural-andbiologicalsciences/plant- diseases

3. Plant Disease Detection Using Neural Network: A REVIEW JETIR1902C27 Journal of Emerging Technologies and Innovative Research (JETIR) www.jetir.org 151

4. Gibson, D., Burghardt, T., Campbell, N., and Canagarajah, N. (2015). "Towards automating visual in-field monitoring of crop health," in IEEE International Conference on Image Processing (ICIP), 2015 (Quebec City, QC: IEEE), 3906-3910.

5. Siricharoen, P., Scotney, B.,Morrow, P., and Parr, G. (2016). "A lightweight mobile system for crop disease diagnosis," in International Conference Image Analysis and Recognition, (Póvoa de Varzim: Springer), 783-791.

6. Dubey, S. R., and Jalal, A. S. (2014). Adapted approach for fruit disease identification using images. arXiv preprint arXiv:1405.4930

7. Mohanty, S. P., Hughes, D. P., and Salathé, M. (2016). Using deep learning for image-based plant disease detection. Front. Plant Sci. 7:1419. doi: 10.3389/fpls.2016.01419

8. Open CV Based Disease Identification of Mango Leaves Jayaprakash Sethupathy \#1, Amrita Vishwa Vidyapeetham, Amrita Coimbatore, India 6410351 s_veni@cb.amrita.edu

9. Szegedy, C., Vanhoucke, V., Ioffe, S., Shlens, J., andWojna, Z. (2016). "Rethinking the inception architecture for computer vision," in Proceedings of the IEEE Conference on Computer Vision and Pattern Recognition (Las Vegas), 2818-2826.

10. Karpathy, A., Toderici, G., Shetty, S., Leung, T., Sukthankar, R., and Fei- Fei, L. (2014). "Large-scale video classification with convolutional neural networks," in Proceedings of the IEEE conference on Computer Vision and Pattern ecognition (Columbus), 1725-1732. 